\title{
Synthetic Image Modalities Generated from Matched CT and MRI Data: A New Approach for Using MRI in Brachytherapy!
}

\author{
R. Krempien ${ }^{1}$, H. A. Grabowski ${ }^{2}$, W. Harms ${ }^{1}$, F. W. Hensley ${ }^{1}$, S. Hassfeld ${ }^{3}$, \\ U. Mende ${ }^{1}$, M. Treiber ${ }^{1}$, M. Wannenmacher ${ }^{1}$ \\ ${ }^{1}$ Clinic for Radiology, Department of Clinical Radiology \\ University of Heidelberg, INF 400, 69120 Heidelberg, Germany \\ ${ }^{2}$ Institute for Process Control and Robotics, Department of Computer Science \\ University of Karlsruhe, 76128 Karlsruhe, Germany \\ ${ }^{3}$ Clinic for Cranio-Maxillo-Facial Surgery \\ University of Heidelberg, INF 400, 69120 Heidelberg, Germany \\ robert_krempien@med.uni-heidelberg.de
}

\begin{abstract}
Target definition and dosimetric evaluation of brachytherapy procedures is crucial in developing proper technique and has prognostic implications. Accurate definition of tumour volume and organs at risk is essential for treatment planing. CT accurately localise sources, but often poorly delineates tumour volume. Magnetic resonance imaging (MRI) has introduced several imaging benefits such as improved soft tissue definition and unrestricted multiplanar and volumetric imaging. However, MRI has not yet seriously challenged CT for brachytherapy planing in most sites. One main reasons for this is the poor imaging of the applicator dummy markers required for detecting the source dwell positions. Therefore a new system generating synthetic image modalities from matched and fused CT and MRI-data has been developed. The system presented consists of five modules serving for viewing, matching, segmentation, fusion and validation. With the presented new system manual or automatic image registration and fusion of CT and MR-images can be done, enabling the use of the imaging advantages of MRI and CT in brachytherapy planing. Tumour structures or source dwell positions could be measured in any plane in one imaging modality and then copied into the other. With synthetic image-based brachytherapy planing a optimisation of target volume covering and dose distribution to treatment volume and structures at risk could be achieved. These preliminary data show that image registration and fusion is feasible for brachytherapy planing. The procedure integrating synthetic images from CT and MRI into brachytherapy planing has the advantage of precise target volume definition and of better determination of dose within this target taking into account the critical dose at structures at risk. Synthetic images may lead to an improved tumour control and reduced side effects by brachytherapy.
\end{abstract}

\footnotetext{
${ }^{1}$ The work is partly being funded by the 'Sonderforschungsbereich Informationstechnik in der Medizin Rechner- und sensorgestütze Chirurgie' of the Deutsche Forschungsgemeinschaft
} 


\section{Introduction}

Brachytherapy from the Greek word "brachy - short distance" consists of placing sealed radioactive sources close or in contact with the target tissue. Implantation techniques may be broadly characterised in terms of surgical approach, i.e. interstitial or intracavitary. In modern brachytherapy using afterloading techniques a single stepping radioactive source is introduced into applicators or guides according to a chosen plan with a predetermined geometrical distribution of possible source dwell positions. Individual adjustment of dose distribution in the target volume and structures at risk can be performed by controlling the dwell time used at each dwell position. Before inserting radioactive sources a dummy marking chain representing the source dwell positions is introduced. Methods using radiographs or computed tomography (CT) accurately localise sources [7]. Computerised dosimetry generally has been generated from orthogonal radiographs rendering limited information about spatial relationship between source positions and tumour volume or structures at risk. The CT-based reconstruction allows the individual adjustment and optimisation of the dose distribution to anatomical target volumes $[4,16]$. But due to the poor soft tissue contrast CT still has limitations in determing target volume and structures at risk [3]. Magnetic resonance imaging (MRI) has introduced several added imaging benefits that may confer an advantage over the use of CT in brachytherapy planing such as improved soft tissue definition and unrestricted multiplanar and volumetric imaging [9]. However, MRI has not yet seriously challenged CT for brachytherapy planing in most sites. The reasons for this include (1) the poor imaging of bone and the lack of electron density information from MRI required for dosimetric calculations; (2) the poor imaging of the applicator dummy required for detecting the source positions due to the lack of source visibility (3) the presence of intrinsic system-related and object-induced MR image distortions [3,9,17].

Synthetic image modalities from matched and fused CT and MRI-data may add the advantages of both imaging modalities [8]. The registration and fusion of medical images, especially the registration of CT and MR images, may be a useful step for brachytherapy planing [12]. The aim of this study was to access the possibilities of synthetic images from registered and fused CT- and MR-Images in brachytherapy planing.

\section{Materials and Methods}

\subsection{Patients and Brachytherapy}

In 1998 two Patients (one Patients with cervix carcinoma, one Patients with soft tissue sarcoma) receiving a combined therapy of external beam and brachytherapy have been planned in parallel mode using (1) CT-based brachytherapy planing and (2) brachytherapy planing based on synthetic images of both imaging modalities (CT 
and MRI). Target volume, treatment volume, planing volume and the resulting optimised dose distribution was calculated. The dose distribution in structures at risk was also calculated. This was performed by two radiation therapists and one physicist. The brachytherapy planing was performed at the planing system Plato (Nucletron, Netherlands). The patients were either treated with high dose rate (HDR) or pulsed dose rate (PDR) brachytherapy.

\subsection{Image Acquisition}

After positioning of brachytherapy applicators or surgical implantation of brachytherapy guides CT and MRI was performed. Since the examination with a brachytherapy applicator needs a larger variety in patient positioning MRI was performed using a open low field MRI (Picker Outlook 0.23 Tesla). T1-weighted spinechoes with and without Gadolinium and T2-weighted spin-echoes were used. The geometric distortion of the MRI-facility was investigated by a special phantom using spin-echo sequences. Within $10 \mathrm{~cm}$ around the isocentre - the region of interest for brachytherapy-planing- this distortion was below $1 \mathrm{~mm}$ [13].

\subsection{System Overview}

For interaction with medical images a system is required which guides the user through several image processing tasks. For that reason a system consisting of five modules was developed. At each time during the image processing task the obtained results can be validated and, if necessary, the task can be repeated.

Viewing: At the beginning of a session, both volumetric data are loaded with the viewer module. This module is designed to exploit as much information as possible from the original slices. In two adjacent windows, the MR and CT images can be visualised in arbitrary planes, the slices can be interpolated, and a histogram-based grey value normalisation can be performed. Other features like picking grey values or cutting regions are available.

Matching: In this module, the images can be registered manually or automatically. The automatic registration takes about one to three minutes for two data sets of about 60 slices. The matched images can be visualised in checkerboard overlay. The automatic registration algorithm is able to register MRI and CT data in over 95 percent of the cases without any interaction. Depending on slice thickness the achieved average accuracy of the calculated transformation was bellow 1 millimetre [11].

Segmentation: In this module, the images can be segmented. Global thresholding and region-growing based seed-point segmentation is available. Up to 15 different structures can be segmented with an index-structure, so that one voxel can belong to two or more structures. Logical operations like (AND, OR, etc.) can be performed with the structures. All segmented structures can be visualised with different volume 
rendering techniques, like gradient shading, MIP (Maximum Intensity Projection) or SIP (Summarised Intensity Projection). The module consists of two modes, the volume mode and the slice mode. In the volume mode three dimensional region growing can be used to segment connected structures. Since different structures are sometimes connected via thin regions, the size of the seed point can be chosen. In the slice mode fine segmentation can be performed slice by slice. For segmentation, polygon lines are used. The previously registered images are superimposed in one window, while it is possible to blend smoothly between the two images. In the superimposed image, interactive segmentation can be performed slice by slice.

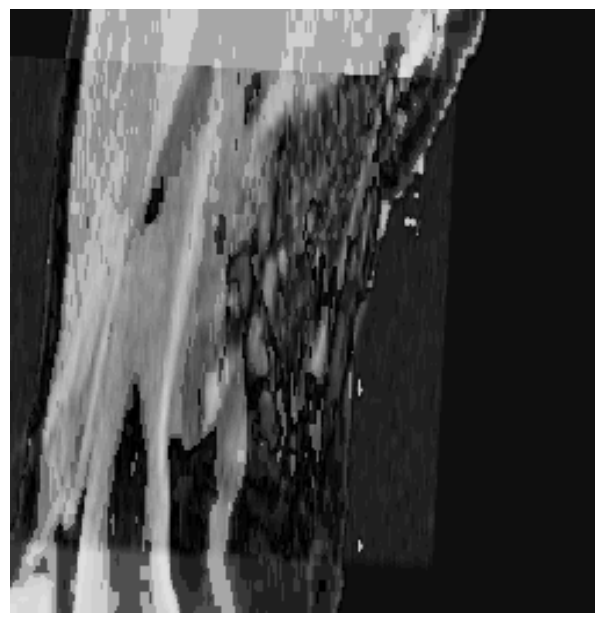

Fig. 1. The MR image and the CT image are fused by the fusion function addition (The grey values of both images are added after a certain kind of grey-value adaptation). Since bony structures are represented with high grey values in CT images, while these structures - giving no MRI-signal in T1w- and T2w-images - are represented with low grey values in MR images the result of the addition of both grey values can not exceed a certain threshold. Otherwise a high grey value in CT images corresponds to a high value in MR images which indicates an error in registration at the bony structures. As seen in the above image the bony structures and the skin contour fit well. Reconstruction of the fused images in different planes offer the possibility to validate the matching accuracy. The result of the fusion obtained by multiplication, addition and subtraction can also be used as a new, synthetic modality.

Validation and Fusion: The grey values of the segmented structures can be copied and pasted from one volumetric image into the other (Fig. 4). In order to obtain high accuracy, the segmented regions are re-sampled before insertion. To avoid aliasing effects, the resampling can be performed with different rates. Additionally, the segmented regions can be copied and pasted without copying the grey values, thus enhancing the possibilities of the segmentation task. With the help of that technique, new imaging modalities can be created by a various fusion functions, like addition, 
subtraction, multiplication of grey values The fusion function can be used for validation (Fig. 1). In the superimposition of the images under different fusion functions, the overall fitness of the obtained matching can be visualised transparently. Also technical validation like the average mean square distance between corresponding landmarks is possible

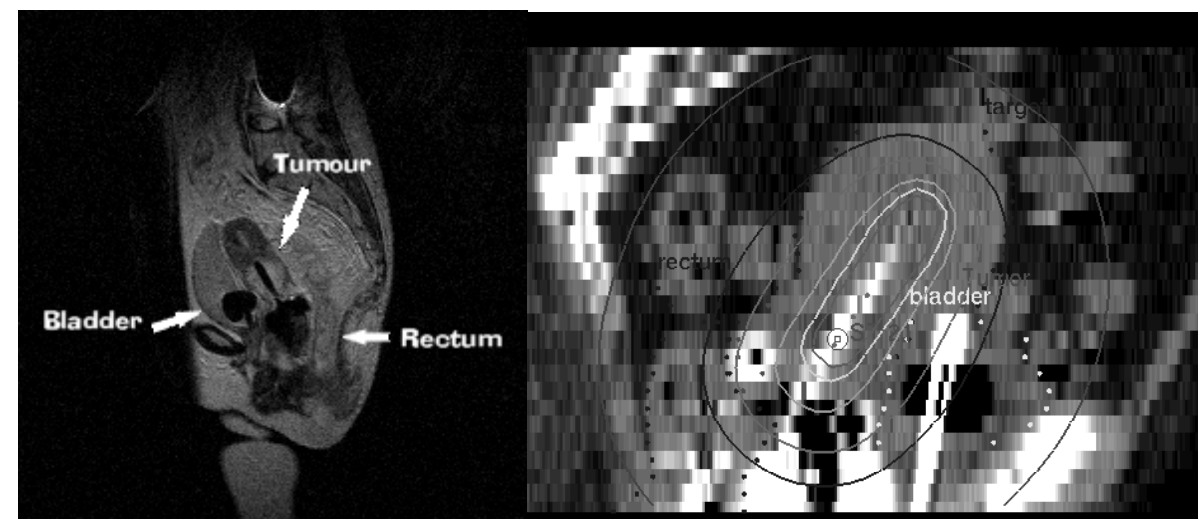

Fig. 2. Cancer of the uterine cervix. Right: Sagittal reconstruction of a pelvic CT-scan with brachytherapy applicator in position. In the planing CT the exact tumour localisation and spread and its relationship to the surrounding structures at risk (i.e. rectum, bladder) were very difficult to determine. Thus brachytherapy planing on $\mathrm{CT}$ alone may result in miscalculation in target definition and dosimetry within this target. Left. In the sagittal pelvic MRI scan the tumour localisation and the relationship to the surrounding tissue is easily visible. The combination of both imaging modalities makes dosimetric optimisation possible with respect to the tumour and the structures at risk.

\section{Results}

The described system for image registration and fusion can (1) visualise and reconstruct CT and MRI- images in any desired plane (2) manually or automatically register CT- and MRI-images. (3) segment multiple structures using global thresholding and region-growing based seed-point segmentation (4) superimpose the previously registered images and segment manually or automatically structures slice by slice (5) transfer the grey volumes of the segmented structures from one volumetric image into the other and (6) validate the achieved accuracy of the matching and fusion procedure interactively. The automatic registration process takes about one to three minutes for two data sets of about 60 slices. The algorithm is able to register MRI and CT data in over 95 percent of the cases without any interaction. The average accuracy of the calculated transformation was below 1 millimetre [11].

With the help of the information of both imaging modalities the optimal imaging plane for further brachytherapy planing could be chosen (Fig. 2). After registration 
structures of interest could be segmented in either one of the imaging modalities. Since MRI has a superior soft tissue contrast possible tumour structures, target structures or organs at risk were segmented in the MRI-data. The applicator dummy markers which were not visible in the MRI could only be segmented in the CT (Fig. 3). The image fusion offered the possibility to merge both information in one image (Fig. 4 left). Since the brachytherapy planing system can only work with Diacom3 data the grey values of the segmented structures from one imaging modality were copied and pasted to the other. These generated synthetic images were retransformed in Diacom3 and imported into the brachytherapy planing system for further planning. The use of the synthetic images leads to a significant adjustment and optimisation of the dose distribution to target volumes and structures at risk.
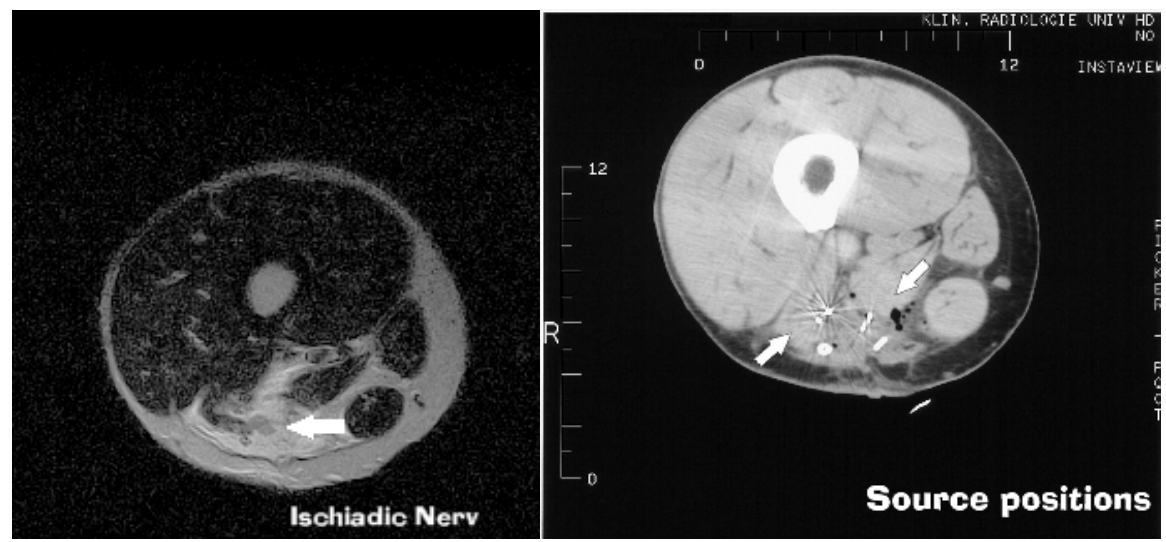

Fig. 3. A patient with liposarcoma in the thigh showed a histological positive tumour margin to the ischiadic nerve. Intraoperativley the decision was made to implant brachytherapy guides for postoperative boost-radiation of the tumour-bed rather than resecting the nerve to preserve the function of the leg. CT (right) accurately localises source positions (arrows) but due to the limited soft tissue contrast and the postoperative changes the nerve as the primary target structure was hardly detectable. The MRI (left) with its superior soft tissue contrast easily showed the nerve (arrow) but no source positions could be visualised.

For example a patient with liposarcoma in the thigh showed a positive tumour margin to the ischiadic nerve. Since further operating meant to resect the nerve which would have resulted in a paralysed leg the intraoperative decision was made to implant brachytherapy-guides for postoperative boost-radiation of the tumour-bed. The nerve as the primary target structure was hardly detectable in the planning CT alone not only due to the poor soft tissue contrast but due to the postoperative changes. In the MRI the nerve could be easily located and it was possible to segment it precisely (Fig. 3). Since both imaging modalities had been registered it was possible to copy the grey values of the segmented nerve into the planing CT. With the help of these generated synthetic images the nerve and its relationship to the source dwell positions could be 
determined in one image set (Fig. 4 left). Based on the synthetic images a new dose prescription was applied (Fig. 4 right). The comparison of the CT-based and synthetic image based planing revealed that the nerve as the primary target structure was incompletely covered or heavily overdosed in $30 \%$ of the target volume based on CTplaning alone. This could have resulted in early tumour relapse or excessive radiation damage to the nerve. The synthetic image based planing resulted in superior target definition and a determination of dose within this target taking into account the critical doses at structures at risk (Fig. 4). Since there was no predefined correct object to compare against various fusion function like addition, subtraction, multiplication of grey values of the registered images were generated in the validation mode. These fused images were used to validate the matching accuracy comparing different anatomical structures i.e. bone and skin contour. As shown in Figure 1 a good matching result could be achieved.

\section{Discussion}

Brachytherapy offers two main advantages in radiation therapy [1]: (1) The radioactive source can be applied close to or into the tumour volume, sparing the surrounding tissue which in external beam radiation often limits the dose escalation needed for tumour control. (2) The steep dose gradient (Fig. 2, 4) enables the delivery of radiation doses into the target volume while sparing structures at risk. As shown in Figure 4 a small error in target volume definition can lead to an incorrect enclosure of tumour volume or to a overdose to organs at risk. This may lead to early tumour relapse and severe side effects [14]. Having this in mind the necessity for exact definition of target volume is clearly visible.

Modern brachytherapy using afterloading technique offers the possibility of individual target definition. Since CT is bound to axial scanning and offers limited soft tissue contrast, it is sometimes difficult to determine the exact tumour location and spread [3]. MRI has introduced several added imaging benefits that may confer an advantage over the use of CT in brachytherapy planing. The problem with MRI in brachytherapy-planing is despite the presence of intrinsic system-related and objectinduced MR image distortions that MRI cannot visualise the dummy source positions required for detecting the source dwell positions needed for further planing [3,9].

Experiments with contrast agent (i.e. gadolinium) filled applicator guides did not result in satisfactory determination of source positions $[6,10]$. One method of avoiding the problems associated with MRI is to integrate the superior tumour definition of MRI with the accurate source localisation, the electron density information and the geometric accuracy obtained from CT. This allows for the integration of comprehensive anatomical mapping with functional distribution of the tissues within a single image set [8]. The registration of medical images, especially the registration of CT and MR images, may be a useful step for brachytherapy planing [12]. Manual methods of registering different images sets can be inefficient and error prone. In manual transposition of target information calculated mismatches of up to $8.5 \mathrm{~mm}$ 
between the volumes outlined by CT compared to MRI have been reported [2]. Having in mind the steep dose gradient this certainly is not acceptable. Automated approaches will reduce inter-operator variability and allow more accurate registration between multimodality systems [15]. Problematic for image registration are the image distortions of the MRI.

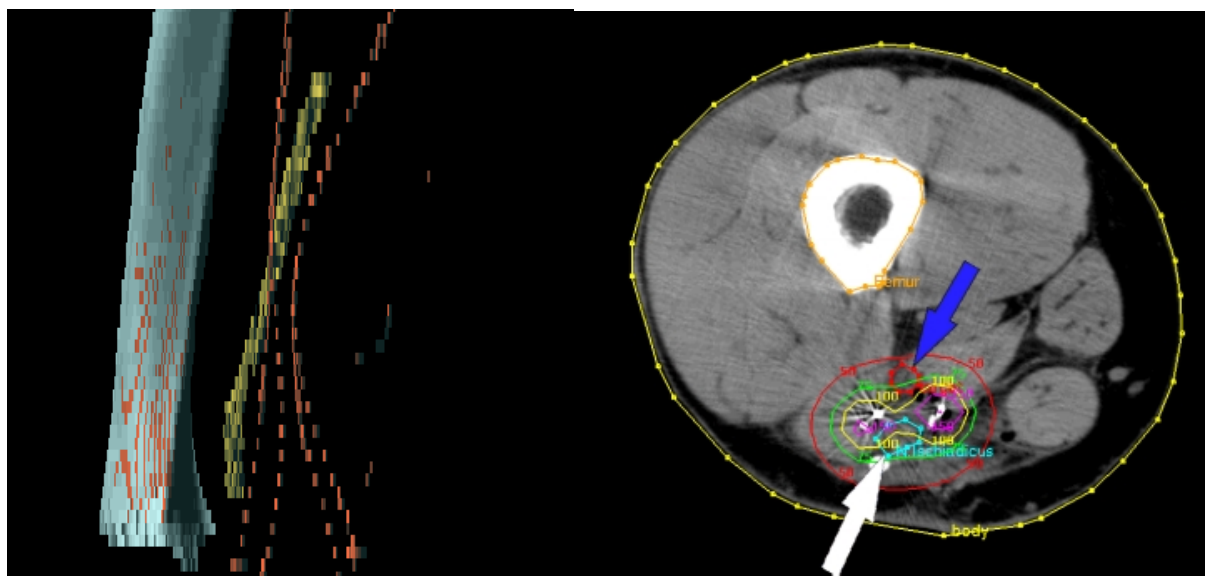

Fig. 4. Left: The ischiadic nerve (yellow structure) is segmented in MRI and the segmented structure can be visualised and positioned in the CT image. The dummy source positions (red) and the femoral bone (blue) are segmented via seed point segmentation in CT. The Position of the nerve can now be visualised in comparison to the source dwell positions. Right: The fused synthetic images are added into the Plato brachytherapy planing system. The red circle (blue arrow) was the supposed nerve localisation due to the planing CT, the turquoise circle (white arrow) due to the planing MRI. The $100 \mathrm{cGy}$ isodose/pulse (yellow line) was the enclosing target volume isodose. The use of a CT alone for brachytherapy-planing would have resulted in a heavy overdose of the nerve with a high risk of radiation damage to the nerve in this slice position, in other places a actual miss of the target structure occurred which may have resulted in early tumour relapse.

Since patient positioning and scanning with brachytherapy applicators or guides sometimes is difficult in a high field MRI due to limited space an open low field MRI was used for the planing MRI. The low field MRI shows less patient induced image distortions since they accumulate with higher magnetic field strengths [17]. But the inhomogeneity in the magnetic field due to the open construction resulted in stronger system induced distortions [17]. The measurement of the geometric distortion investigated by a special phantom showed that within $10 \mathrm{~cm}$ around the isocentre this distortion was below $1 \mathrm{~mm}$ and the phantom investigation offered a possibility to correct these distortions, thus disturbing geometric distortions could be overcome [13]. But distortion of anatomical structures due to patient movements are still problematic. This can result in problems in the automatic registration process, needing manual 
adjustment. The use of deformable image models using finite element meshes [5] may be a possible solution but needs further investigation.

The presented interactive system allows full automatic registration, based on voxel similarity measurements. After segmentation structures in the matched images can be copied from one image into the other. Tumour structures or source dwell positions can be segmented in one imaging modality and then copied into the other thus enabling the use of the superior soft tissue contrast of the MRI in brachytherapy planing. The planing procedure integrating synthetic images from CT and MRI has the advantage of precise target volume definition and of determination of dose within this target taking into account the critical dose at structures at risk.

\section{Conclusion}

These preliminary data show that image registration and fusion is feasible for brachytherapy planing. The procedure integrating synthetic images from CT and MRI into brachytherapy planing has the advantage of precise target volume definition and of better determination of dose within this target taking into account the critical dose at structures at risk. Synthetic images may lead to an improved tumour control and reduced side effects by brachytherapy.

\section{References}

[1] K.S.C. Choa, C.A. Perez, L.W. Brady Radiation Oncology: Managment Decisions. Lippincott - Raven, Philadelphia New York p79-99, 1999

[2] A.W.T. De Valles, M. Abe, R. N: Kjellberg Transposition of target information from magnetic resonance and computed tomograpy scan images to concentional stereotacic space. Appl. Neurophysiol. 50:23-32 1987

[3] D.F. Dubois, B.R. Prestidge, L.A. Hotchkiss, W.S. Bice, J.J. Prete Source localisation following permanent transperineal prostate interstitial brachytherapy using magnetic resonance imaging. Int. J Radiat. Oncol. Biol. Phys. 39:1037-1041;1997

[4] B. Erickson, K. Albano, M. Gillin CT-guided interstitial implantations of gynecologic malignancies. Int. J Radiat. Oncol. Biol. Phys. 36:699-709; 1997

[5] H.A. Grabowski, J. Brief, S. Hassfeld, R. Krempien, J. Raczkowsky, H. Wörn, U. Rembold Model-based registration of medical images using finite element meshes, In: H. Lemke, M.W. Vannier, K. Inamura, A.G. Farman (eds.): Proceedings of the $12^{\text {th }}$ International Symposium and Exhibition on Computer Assisted Radiology and Surgery (CAR'98), Tokyo, Elsevier Press, 1998, p. 159-163

[6] W. Harms, R. Krempien, F. Hensley, M. Wannenmacher. Work in progress: Using MRI in brachytherapy planing

[7] F.W. Hensley, W. Harms, R. Krempien, P. Fritz, Ch. Berns, M. Wannenmacher Analysis of the geometrical accuracy of CT-based interstitial brachytherapy reconstructions. GEC Estro 1999 
[8] K. Kagawa, W.R. Lee, T.E: Schultheiss, M.A. Hunt, A.H. Shaer, G.E. Hanks Initial clinical assessment of CT-MRI image fusion software in localisation of the prostate for $3 D$ conformal radiation therapy. Int. J. Radiation Oncology Biol. Phys. 38:319-325

[9] V.S. Khoo, D.P. Dearnaley, D.J. Finnigan, A Padhani, S.T. Tanner, M.O. Leach: Magnetic resonance imaging (MRI): considerations and applications in radiotherapy treatment planing. Radiother. Oncol. 42:1-15; 1997

[10] G. Kovacs, R. Pötter, F.J. Prott, B. Lenzen, T.H. Knocke The Münster experience with magnetic resonance imaging assisted treatment planing used for high dose rate afterloading therapy of gynaecological and nasopharyngeal cancer. Advanced Radiation Tumour Response. in: Breit (Ed.): Monitoring and Treatment Planing, Springer Heidelberg 1992: p661-665

[11] P. Pokrandt Fast Non-Supervised Matching: A Probabilistic Approach, Proceedings of the $10^{\text {th }}$ International Symposium and Exhibition on Computer Assisted Radiology and Surgery (CAR’96), 1996

[12] J.G. Rosenman, E.P. Miller, G. Tracton, T. Cullip Image registration: an essential part of radiation therapy treatment planing. Int. J. Radiation Oncology Biol. Phys. 40:197-205

[13] K. Schubert, F. Wenz, R. Krempien, O. Schramm, G. Sroka-Perez, P. Schraube, M. Wannenmacher Einsatzmöglichkeiten eines offenen Magnetresonanztomographen in der Therapiesimulation und dreidimensionalen Bestrahlungsplanung. Strahlentherapie Onkologie 175:225-231, 1999

[14] A. Terahara, T. Nakano, A. Ishikawa, S. Morita, H. Tsujii Dose-volume histogram analysis of high dose rate intracavitary brachytherapy for uterine cervix cancer. Int. J. Radiation Oncology Biol. Phys. 35:549-554; 1996

[15] P.A. van den Elsen ;E.J. Pol, M.A. Viergever Medical image matching - a review with classification. IEEE Trans. Med. Biomed. Eng. 12:26-39, 1993

[16] K.J. Weeks, G.S. Montana Three-dimensional applicator system for carcinoma of the uterine cervix. Int. J Radiat. Oncol. Biol. Phys. 37:455-463; 1997

[17] G Wesby, M.K. Adamis, R.R. Edelmann: Artifacts in MRI: description, causes and solutions. In: Edelmann RR, Hesselink JK, Zlatkin MB Eds.: Clinical magnetic resonance imaging. Saunders, Philadelphia, p88-144, 1996 